\title{
Radiation pattern and impurity transport in argon seeded ELMy H-mode discharges in JET
}

\author{
M E Puiatti ${ }^{1}$, M Mattioli ${ }^{1}$, G Telesca ${ }^{1}$, M Valisa $^{1}$, I Coffey $^{2}$, \\ P Dumortier ${ }^{3}$, C Giroud ${ }^{4}$, L C Ingesson ${ }^{5}, \mathrm{~K}$ D Lawson ${ }^{6}$, G Maddison $^{6}$, \\ A M Messiaen ${ }^{3}$, P Monier-Garbet ${ }^{4}$, A Murari ${ }^{1}$, M F F Nave ${ }^{7}, \mathbf{J}_{\text {Ongena }}{ }^{3}$, \\ J Rapp $^{8}$, J Strachan ${ }^{9}$, B Unterberg ${ }^{8}$, M von Hellermann ${ }^{5}$ and \\ contributors to the EFDA-JET Workprogramme ${ }^{10}$
}

${ }^{1}$ Associazione Euratom-ENEA sulla Fusione, Consorzio RFX, Padova, Italy

${ }^{2}$ Queens University, Belfast BT7 INN, North Ireland

${ }^{3}$ LPP-ERM/KMS Euratom-Belgian State Association, Brussels, Belgium

${ }^{4}$ Association Euratom-CEA, DRFC, CEA Cadarache, St Paul lez Durance, France

${ }^{5}$ FOM Instituut voor Plasmafysica Rijnhuizen, EURATOM Association, Nieuwegein, The Netherlands

${ }^{6}$ EURATOM/UKAEA Fusion Association, Culham Science Center, Abingdon, UK

${ }^{7}$ Associacao Euratom-IST, Centro de Fusao Nuclear, Lisboa, Portugal

${ }^{8}$ Institut fur Plasmaphysik, Forschungszentrum Julich GmbH, Euratom Association, Julich, Germany

${ }^{9}$ Princeton Plasma Physics Laboratory, USA

Received 16 January 2002, in final form 9 May 2002

Published 27 August 2002

Online at stacks.iop.org/PPCF/44/1863

\begin{abstract}
This paper addresses the issues of impurity behaviour during the argon seeding experiments in JET, in which argon and $\mathrm{D}_{2}$ have been simultaneously puffed in ELMy H-mode discharges to reach high density regimes, maintaining good confinement properties throughout the plasma discharge. The analysis is based mainly on a 1-D impurity diffusion model, which evaluates the impurity transport coefficients in three experimental scenarios.

The available experimental data include, as a function of the time, the brightnesses of the soft $\mathrm{x}$-rays and of several impurity lines, the effective charge $Z_{\text {eff }}$, the profiles of the radiated power and the fully stripped $\mathrm{Ar}$ and $\mathrm{C}$ ion densities from charge-exchange recombination. From the simulation of these data it is possible to characterize the considered scenarios in terms of high- $Z$ ion increase in the plasma centre and high radiation from the edge. In particular, it has been found that the configuration at high triangularity with continuous $\mathrm{D}_{2}$ puffing, characterized by an outward impurity pinch velocity, features flat or slightly hollow impurity ion profiles and high radiation from a narrow region at the edge, possibly associated to the presence of a significant neutral density. In this case an edge radiative mantle is established without high radiation from the plasma centre.
\end{abstract}

\footnotetext{
${ }^{10}$ Appendix of J Pamela, JET-EFDA Team in Fusion Energy 2000 Proc. 18th Int. Conf. (Sorrento, 2000) (Vienna: IAEA) 2001 CD-Rom file PD/1 (see affiliation 2 also).
} 


\section{Introduction}

Impurity seeding has been used in tokamak devices to produce a poloidally and toroidally uniform radiative mantle in order to obtain a uniform deposition of the input energy (and therefore a reduced thermal load) on the walls. This is an important issue for future reactors, since, with increasing input power, the heating and the erosion of the first wall become a critical point. It has been found in TEXTOR and subsequently in other devices (Asdex-Upgrade, DIII-D, JT-60U) that in these highly radiative regimes the plasma performance improves when density limits are approached (for an overview of these experiments, see [1] and the references quoted there). Of course the accompanying risk is an increase of the plasma pollution, with large values of effective charge $Z_{\text {eff }}$ and of the radiated power density from the plasma centre. A general aim of the impurity seeding experiments is to obtain a high radiation level from the edge avoiding impurity accumulation in the centre.

Impurity seeded ELMy H-mode discharges, featuring simultaneously high density (Greenwald factor $\left.N_{\mathrm{GW}}=n_{\mathrm{e}} / n_{\mathrm{GW}} \approx 1\right)$, high confinement factor $\left(f_{\mathrm{H} 97} \approx 1\right)$ and high edge radiation losses, have been successfully experimented in JET [2]. Ar and $\mathrm{D}_{2}$ are puffed simultaneously in ELMy H-mode discharges to reach high density regimes. During this phase the confinement degrades, but as soon as the gas injection is switched off or it is reduced to quantities adequate to maintain the density (so called after-puff (AP) phase), the good H-mode confinement is recovered.

The highly performing AP phase has been tested in different magnetic configurations, with different heating schemes and with a variety of gas puffing rates. The aim was not only the optimization of the confinement but also of the radiation efficiency and of the power exhaust, and the avoidance of impurity accumulation. It is not surprising that the main difficulty in these experiments was to maintain all these plasma parameters stationary [2].

In this context, a detailed study of Ar diffusion in such JET plasmas has not been done up to now, in particular the accumulation of $\mathrm{Ar}$ in the centre in the different experimental scenarios has to be studied. In fact, the specific motivation of this paper is to characterize different $\mathrm{Ar}$ seeded plasmas with respect to the equilibrium between highly radiative regimes at the edge and increase of high- $Z$ impurity ions in the centre in long lasting discharges. The aim is to identify high performance regimes with hollow radiation profiles and without accumulation of seeding impurity ions in the plasma centre. This study is accomplished by simulating the behaviour of Ar and C line brightnesses, of the SXR emission, of the plasma effective charge and of the radiated power in three different plasma configurations.

The paper is organized as follows. Three experimental scenarios, all with Ar seeding, are analysed and presented in section 2. The first two refer to a low triangularity septum divertor configuration with respectively neutral beam injection (NBI) heating only and $2 \mathrm{MW}$ ion cyclotron resonance heating (ICRH) added to NB. The third experimental scenario refers to a high triangularity discharge with strong continuous $\mathrm{D}_{2}$ puffing, heated by NB plus $1.7 \mathrm{MW}$ of ICRH. Section 3 describes the simulations based on a 1-D time dependent impurity transport code [3]. The code follows the radial distribution of the injected $\mathrm{Ar}$ and intrinsic $\mathrm{C}$ ions. Both line brightnesses and emissivities are obtained. Post-processing subroutines evaluate the radiated power, the soft $x$-ray (SXR) signals and the $Z$-meter visible continuum bremsstrahlung emission. Comparing the results of the simulations with the experimental data, it is possible to obtain the $\mathrm{Ar}$ and $\mathrm{C}$ distributions as well as their transport coefficients, namely a diffusion coefficient $D$ and a convection velocity $v$. In $[3,4]$, respectively, $n=2$ to $n=3$ and $n=2$ to $n=2$ L-shell Ar spectra were simulated. Ar spectra measured in one of the considered discharges are compared in section 4 with the simulated spectra. From these simulations it is possible to obtain the impurity transport coefficients in the peripheral region just inside the last closed flux surface (LCFS). Finally section 5 contains the concluding remarks. 


\section{Experiments}

As previously mentioned, three experimental scenarios, all performed with $B_{\mathrm{t}}=2.4 \mathrm{~T}$, $I_{\mathrm{p}}=2.5 \mathrm{MA}, q_{95}=3.05$, will be discussed in this paper. The first and the second ones (discharges \#52136 and \#53015, respectively) refer to a septum configuration, i.e. the X-point is on the dome of the divertor, at low triangularity (lower triangularity $\delta_{1}=0.24$, upper triangularity $\delta_{\mathrm{u}}=0.18$ ). The third (discharge \#53548) is at very high triangularity $\left(\delta_{1}=0.35\right.$, $\delta_{\mathrm{u}}=0.45$ ) with the X-point above the septum. The time evolution of the main parameters (indicated in the caption) are reported in figure 1 $(a)$, where the time is referred to the beginning of the AP phase. In these discharges Ar was puffed from the top of the machine.

In the first discharge (\#52136) the heating power from NBI is $12 \mathrm{MW}$. After a phase with strong $\mathrm{D}_{2}$ and Ar puffing, in which the confinement decreases, both $\mathrm{D}_{2}$ and $\mathrm{Ar}$ are puffed as small 'blips' to maintain the density (AP phase), and the confinement factor $f_{\mathrm{H} 97}$ recovers values of about 1 , with $N_{\mathrm{GW}} \geqslant 0.8$ (slightly decreasing) and a total radiation fraction $\gamma_{\mathrm{RAD}}=P_{\text {rad }} / P_{\text {input }} \sim 45 \%$ (where $P_{\text {rad }}$ includes the radiation from the divertor).

Discharge \#53015 is part of an experiment in which 2 MW of ICRH power has been added on top of $12 \mathrm{MW}$ of NBI power, to keep the safety factor $q(0)$ just below 1 , thus maintaining the sawtooth activity with the aim of controlling impurity profiles via the expulsion processes associated to the sawtooth crashes [5,6]. A quasi-stationary AP phase is reached, during which both high density $\left(N_{\mathrm{GW}} \sim 0.85\right)$ and high confinement $\left(f_{\mathrm{H} 97} \sim 1\right)$ at a $\gamma_{\mathrm{RAD}}$ level comparable with that of shot \#52136 are achieved. Comparing shots \#52136 and \#53015, it must be observed that they have different fluxes of deuterium and argon: while in \#52136 during the AP both argon and deuterium are injected as 'blips', in \#53015 in the AP the deuterium flux is stopped and a low Ar flux is continuously puffed, with an overall injected level higher than in the discharge without ICRH. This may be an explanation of the slight increase of the effective charge $Z_{\text {eff }}$ in pulse \#53015.

In discharge \#53548 the heating power is $14 \mathrm{MW}$ from NBI and $1.7 \mathrm{MW}$ from ICRH. Strictly speaking in this case an AP phase cannot be defined, since $\mathrm{D}_{2}$ is continuously puffed throughout the whole discharge. Nevertheless, in the following, for this discharge we will refer to an AP phase as to the phase during which the $\mathrm{D}_{2}$ puffing is slowly decreased and Ar is injected as 'blips'. It has to be mentioned that in this discharge the high triangularity allows the preservation of high confinement also with high $\mathrm{D}_{2}$ puffing: in fact when the $\mathrm{Ar}$ injection starts, the $\gamma_{\mathrm{RAD}}$ level increases up to $\sim 60 \%$, with $N_{\mathrm{GW}}$ also increasing up to 1 in $3 \mathrm{~s}$ at $f_{\mathrm{H} 97} \sim 0.8$ (figure 1 right).

Figure $1(b)$ shows examples of electron temperature and electron density profiles, during the central AP phase for the three analysed discharges to be used in the following simulations. They have been obtained as fit to the Thomson scattering and electron cyclotron emission data, including those from the peripheral scattering system. The temperature profiles are very similar, while the density profile is flatter in the high triangularity discharge.

\section{Impurity and radiation behaviour}

\subsection{The impurity transport model}

The impurity transport code describes in cylindrical geometry ionization, recombination and radial transport of the ions of charge $Z$ according to:

$$
\frac{\partial n_{Z}}{\partial t}=-\frac{1}{r} \frac{\partial\left(r \Gamma_{Z}\right)}{\partial r}+n_{\mathrm{e}}\left(n_{Z-1} S_{Z-1}-n_{Z} S_{Z}+n_{Z+1} \alpha_{Z+1}-n_{Z} \alpha_{Z}\right),
$$




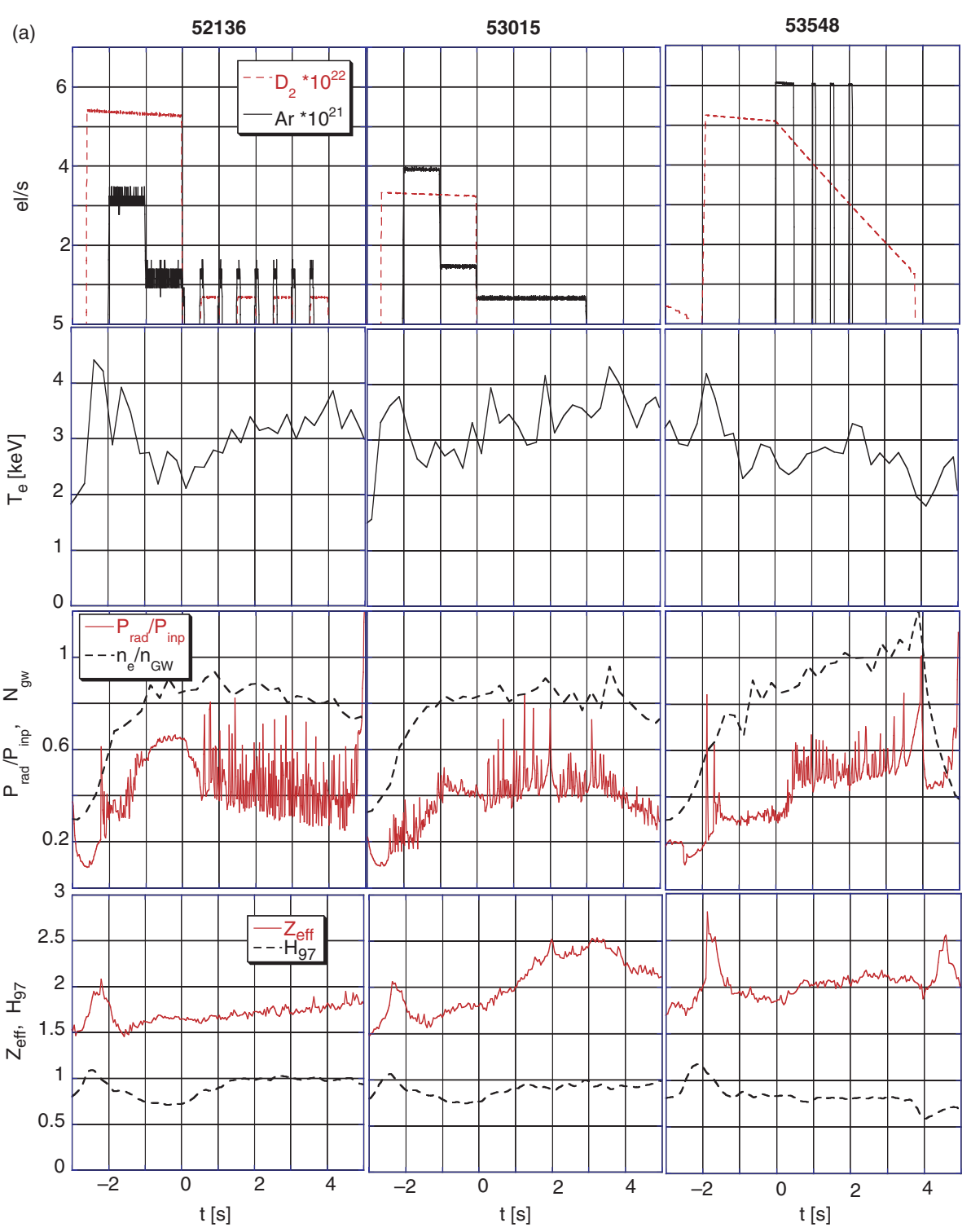

Figure 1. (a) Main plasma parameters of the three discharges analysed in the paper (from left to right): low triangularity septum pulse \#52136 (NBI $12 \mathrm{MW}$ ); low triangularity septum pulse \#53015 (12 MW NBI, 2 MW ICRH); high triangularity pulse \#53548 (14 MW NBI, 1.7 MW ICRH and continuous $\mathrm{D}_{2}$ puffing). The signals (from top to bottom) are: plot $1-\mathrm{D}_{2}$ flux normalized to $10^{22} \mathrm{el} \mathrm{s}^{-1}\left(-\right.$ - $\left.^{-}\right)$and Ar flux normalized to $10^{21} \mathrm{el} \mathrm{s}^{-1}$ (—); plot 2-on-axis electron temperature; plot 3- $\gamma_{\mathrm{RAD}}(-)$ and Greenwald factor $\left.N_{\mathrm{GW}}=n_{\mathrm{e}} / n_{\mathrm{GW}}(--)^{-}\right)$; plot 4-plasma effective charge $Z_{\text {eff }}(-)$ and confinement factor $f_{\mathrm{H} 97}(--)_{-}$. Times are referred to the start of the AP phase. (b) Electron temperature and electron density profiles for the same discharges at $t=2 \mathrm{~s}$.

where the radial ion flux $\Gamma_{Z}$ is expressed in terms of a diffusion coefficient $D$ and a pinch velocity $v$ ( $v>0$ corresponds to outward velocity), assumed independent of the ion charge:

$$
\Gamma_{Z}(r)=-D(r) \frac{\partial n_{Z}(r)}{\partial r}+v(r) n_{Z}(r)
$$



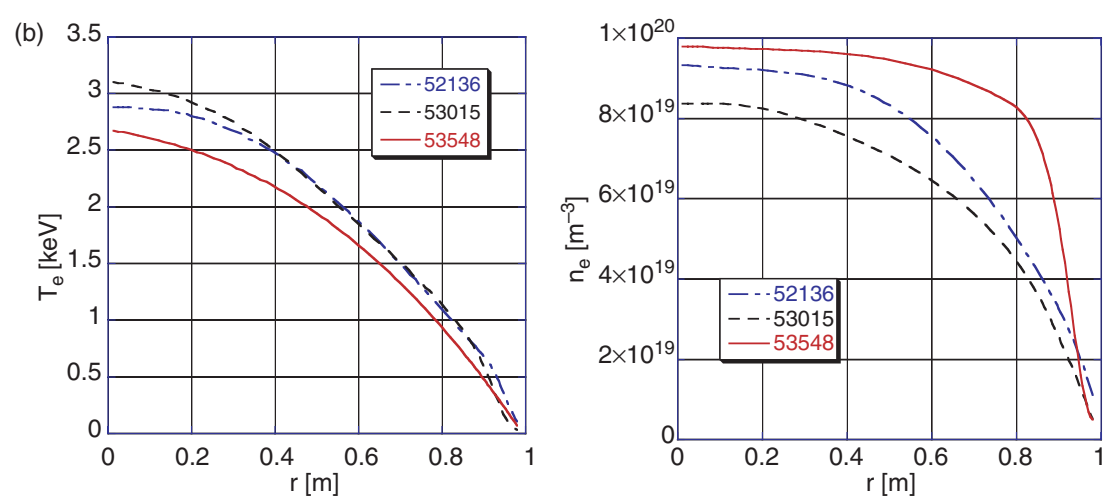

Figure 1. (Continued)

The atomic coefficients in (1), where $S_{Z}$ are the ionization rates for the ions of charge $Z$ and $\alpha_{Z}$ are the radiative plus dielectronic plus charge-exchange recombination rates, have been discussed in $[3,4]$; they depend on the electron temperature and density profiles, input data to the code. The impurity influx is given as boundary condition, supposing that impurities enter the plasma at the last mesh $(\sim 5 \mathrm{~cm}$ outside the LCFS) as neutrals with a velocity of $5 \times 10^{3} \mathrm{~m} \mathrm{~s}^{-1}$ corresponding to $5 \mathrm{eV}$ for Ar. However, this value has negligible influence on the simulation. The time evolution of the influx is determined by tracking the peripheral line brightness time evolutions.

The JET elliptical plasma shape is taken into account as usual by replacing $r$ in the diffusion term with the well-known $V^{\prime}$ function $\left(V^{\prime}=\mathrm{d} V_{\mathrm{p}}(r) / \mathrm{d} r\right.$, where $V_{\mathrm{p}}(r)$ is the volume inside the magnetic surface of radius $r$ ).

The transport coefficients $D$ and $v$ are radius and time dependent and are chosen in such a way to obtain the best ' $g$ lobal' simulation of the available experimental data. $D$, that as a result of the simulations can be taken practically independent of time, is mainly sensitive to the inflows of $\mathrm{Ar}$ (following the valve opening) and of $\mathrm{C}$ (following the application of NBI). Its profile has been found to decrease towards the plasma centre, but it has not been possible to prove the existence of an improved confinement core region, as reported in [6-8] in several JET experimental conditions. To obtain a better $D(r)$ evaluation, Abel-inverted soft x-ray emissivity profiles are necessary (work is in progress in this direction). On the other hand, $v$ is varying in time, starting from the beginning of the AP phase, as required to follow the time evolution of the experimental data depending on the plasma core conditions. Contrary to $D$, which is the same for $\mathrm{C}$ and $\mathrm{Ar}, v$ is different for these two elements, since the fully stripped $\mathrm{C}$ ion density profiles measured by charge exchange recombination (CXR) spectroscopy are always hollow. The determination of the transport coefficients in the LCFS region is obtained from the spectrum analysis discussed in section 4 . As for the simulation of the data reported in $[3,4]$, the analysis points to the presence of a peripheral barrier (either convective or diffusive) in all the three considered scenarios. From the simulation of the spectra in the 23-41 $\AA$ range including both $\mathrm{Ar}$ and $\mathrm{C}$ lines, it is possible to get the relative $\mathrm{C}$ content near the LCFS.

\subsection{Low triangularity septum discharge with $12 \mathrm{MW} \mathrm{NBI}$}

Despite the good confinement and the relatively low effective charge, in shot \#52136 the Abelinverted radiation profiles show a peaking of the radiated power density during the AP phase, as drawn in figure 2 (all the Abel inversions reported in the paper are averaged over $100 \mathrm{~ms}$ ). 


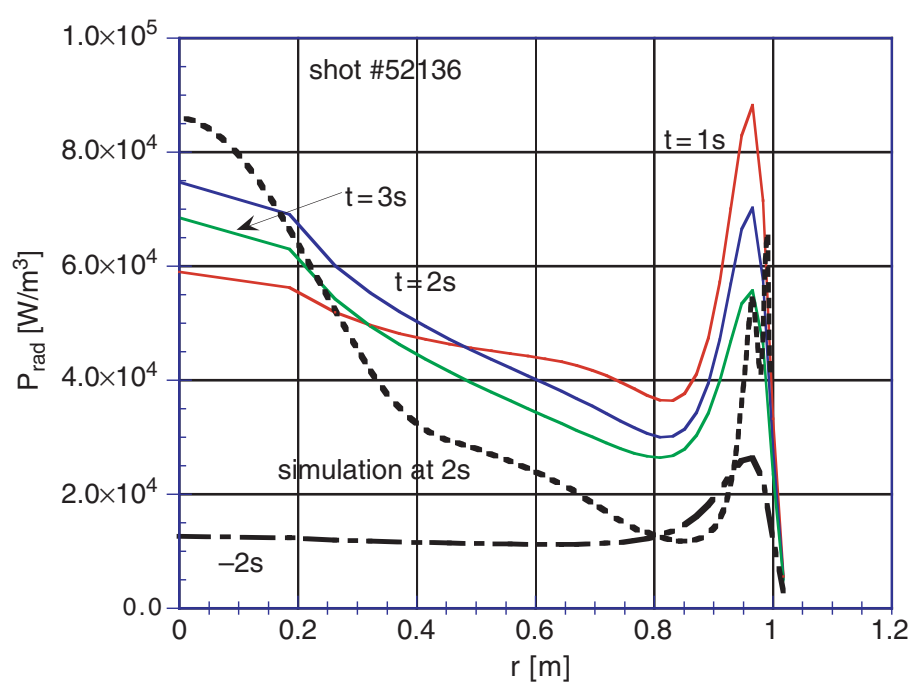

Figure 2. Abel-inverted profiles of the radiated power density before the Ar injection (at $t=-2 \mathrm{~s}$, - - -) and during the AP phase for pulse \#52136, showing a strong peaking ( - ). Times are referred to the start of the AP phase. The result obtained from the simulation at $2 \mathrm{~s}$ is also shown (- - -).

This indicates that an edge radiative mantle without central radiation is not obtained. On the other hand, both the soft x-rays signals, with dominating contribution from central highly charged ions, and the central density of fully stripped $\mathrm{Ar}^{18+}$ ions (from CXR spectroscopy) increase strongly during the AP phase. At the same time the intensities of the Ar XVI and Ar xv lines (measured by a Schwob-Fraenkel extreme grazing incidence spectrometer [9] and by a VUV survey spectrometer Spred [10]) that, due to their ionization potential $<1 \mathrm{keV}$, emit from a more external region, decrease in time. This experimental scenario corresponds to an accumulation of argon (accumulation means Ar density, but not necessarily $\mathrm{C}$ density, increasing in the plasma centre).

To simulate the impurity behaviour, starting from the beginning of the AP phase, an inward pinch velocity must be assumed in the central region. It increases in time during the AP phase and its profile at $t=2 \mathrm{~s}$ after the beginning of the AP is shown by the solid line in figure 3 . As already said in the previous paragraph, the diffusion coefficient, kept constant during the AP phase (dashed line in the same figure), decreases towards the plasma centre. In figure 4 the peaked Ar ion density distribution (always at $t=2 \mathrm{~s}$ ) is shown along with the comparison, at the same time, with the fully stripped $\mathrm{Ar}^{18+}$ ion density from CXR spectroscopy.

The behaviour of carbon, the only intrinsic impurity with large brightnesses, is different from that of Ar: in this case the CX data give hollow profiles of the fully stripped ion density; therefore, in the simulation an outward velocity is assumed in the central plasma region. Also here an edge diffusion barrier is necessary to approach the high experimental value of the ratio $\rho$ between the Ly $\alpha 33.7 \AA \mathrm{C}$ vi brightness and the resonance $40.2 \AA \mathrm{C}$ v line $(\rho \sim 4)$. Contrary to the Ar brightnesses, these $\mathrm{C}$ line brightnesses do not decrease during the AP phase. However, the carbon contribution to the radiated power is small: the Abel inverted profiles before the Ar influx show very low levels of radiation, negligible in the centre and quite low also at the edge, as for example reported in figure $22 \mathrm{~s}$ before the beginning of the AP phase and a few hundreds ms before the Ar injection.

Summarizing, the chosen profiles of the transport coefficients allow the reconstruction of 


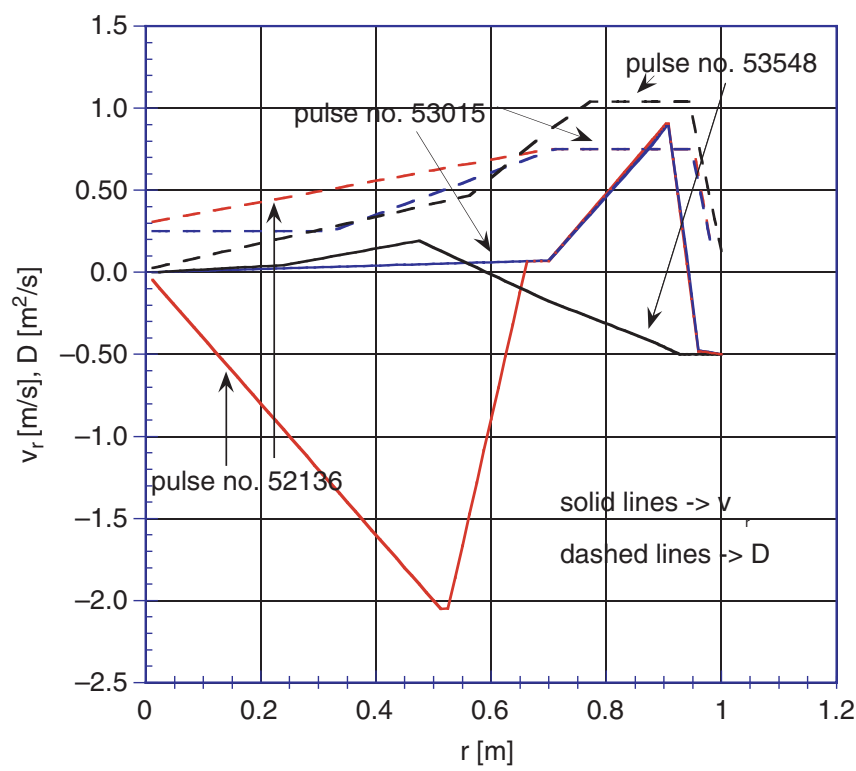

Figure 3. For the three analysed discharges and $2 \mathrm{~s}$ after the beginning of the AP phase (see in the text its definition for \#53548) profiles of the diffusion coefficients (- - - ) and pinch velocities (—). Positive values for $v$ correspond to an outward velocity.

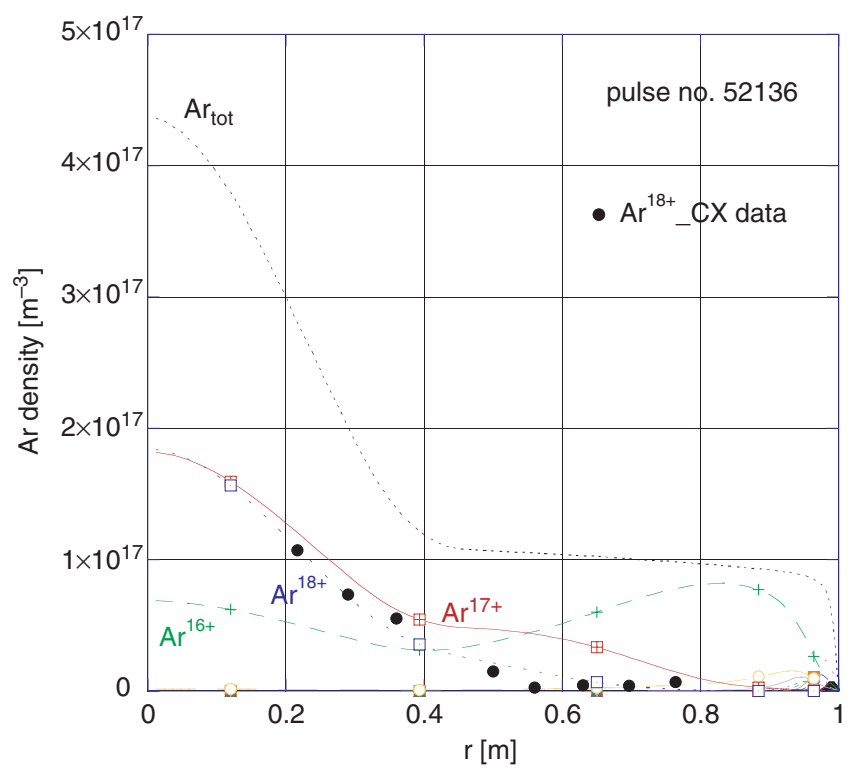

Figure 4. Radial profiles of the Ar ion densities and of the total Ar density obtained from the simulation of pulse \#52136 at $t=2 \mathrm{~s}$ after the start of the AP phase. The experimental points show the $\mathrm{Ar}^{18+}$ ion density obtained from CXR spectroscopy.

the time evolutions of the Ar XVI and Ar Xv line brightnesses, of the central soft X-ray brightness, of the plasma effective charge $(\sim 1.8)$, of the brightnesses of the two indicated $\mathrm{C}$ lines and, though with more uncertainty, of the total radiation profiles. As an example, figure 2 reports 
the simulation obtained at $2 \mathrm{~s}$ after the start of the AP phase for the radiated power density; the agreement between the inversion and the simulation can be considered sufficient, due to the symmetry hyopthesis of the Abel inversion procedure and to the cylindrical symmetry assumed by the simulation model. It has also to be recalled that the power densities shown in figure 2 are obtained neglecting the divertor contribution to the radiated power.

To conclude, we would like to stress again that, to simulate this discharge, variations of $v$, becoming strongly inwards in the central region, are necessary during the enhanced confinement phase ( $f_{\mathrm{H} 97}$ approching 1$)$. Therefore, in this case the AP phase corresponds to high confinement at high density, but obtained in presence of Ar accumulation in the plasma centre.

\subsection{Low triangularity septum discharge with $2 \mathrm{MW}$ ICRH power added to $12 \mathrm{MW} N \mathrm{NBI}$}

The simulation of shot \#53015 requires a different impurity transport scenario. The Abelinverted radiation profiles and the time evolution of the soft x-ray brightnesses do not indicate an impurity accumulation phenomenon as in the previous case. As shown in figure 5, the $P_{\text {rad }}$ profiles remain hollow, with a tendency to peak at about $3 \mathrm{~s}$ after the start of the AP. Nevertheless, it has to be observed that the value of the radiation from the centre is comparable with that observed in the first discharge. The simulation of the impurity behaviour is obtained with essentially the same diffusion coefficient found for the previous discharge, but with a pinch velocity that in the central plasma is very low and outwards in a first phase of the AP (figure 3, at $2 \mathrm{~s}$ ), and in a later phase is slightly inward. The simulated $P_{\text {rad }}$ profile is compared with the experiment in figure 5. Correspondingly, the radial profiles of the Ar ion densities resulting from the simulation give a total Ar density profile slightly hollow or quite flat, as drawn in figure 6 . The simulated value of the line-integrated soft $x$-ray central brightness about $1 \mathrm{~s}$ after the start of the AP phase is $2 \times 10^{4} \mathrm{~W} \mathrm{~m}^{-2}$, to be compared with an experimental value around $3 \times 10^{4} \mathrm{~W} \mathrm{~m}^{-2}$ : the agreement may be considered satisfactory given the uncertainty in the absolute calibration of the diagnostic. The same transport parameters used for argon allow also the simulation of carbon (contrary to \#52136), as expected, since the radial profile of $\mathrm{C}^{6+}$

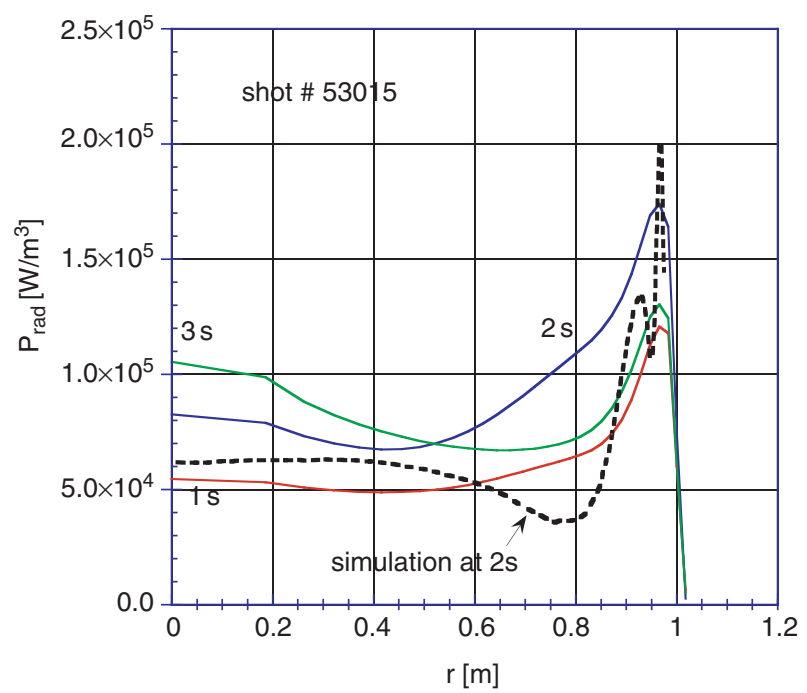

Figure 5. Same as figure 2 during the AP phase in pulse \#53015, where 2 MW ICRH are added to $12 \mathrm{MW}$ NBI. 
(from CX data) is similar to that of the total argon density obtained from the calculation.

In summary, in this case, despite a total Ar influx higher than in the previous discharge, the Ar concentration in the centre results to be lower, while the radiation is higher from the edge and comparable in the centre. This behaviour has been tentatively associated in [5] to the application of ICRH. In fact, the role of ICRH is to maintain sawtoothing, which causes impurity expulsion at crash. This has been in the past already analysed in JET laser blow-off injection experiments reported in [7]. However, even if the sawteeth imply impurity expulsion at crash, their amplitude in this discharge seems insufficient to explain the difference between the global evolution of the Ar ion densities during the AP phases of \#52136 and \#53015. It must also be mentioned that the Ar inflow phases with confinement degradation are quite similar for the two discharges.

The experimental value of $Z_{\text {eff }}$ is about the same in both discharges, but this may be explained considering that the measurement gives a line-of-sight averaged value. Indeed, though similar average values are obtained for the Ar contribution to $Z_{\text {eff }}$, the profile for the first analysed discharge (\#52136) is more peaked than for the latter, as will be drawn later.

One may also speculate that a higher power deposited on the plasma centre would result in a higher on-axis temperature, giving a further benefit in terms of radiation. Indeed, a calculation made with the same transport parameters and density but with a hypothetical on-axis temperature of $6 \mathrm{keV}$ (not corresponding to an experimental situation) predicts that, due to an increased ionization degree of argon (90\% of argon ions would be completely stripped in the centre), the same argon concentration would correspond to a radiation decreasing by a factor of 2 near the axis and remaining about the same at the edge, with a negligible difference in $Z_{\text {eff }}$. This suggests that an experiment with higher ICRH heating power added to NB should be interesting.

The diffusion coefficients and the pinch velocity assumed for the simulation of argon in shot \#53015 have been compared with the predicted neoclassical values. The neoclassical flux

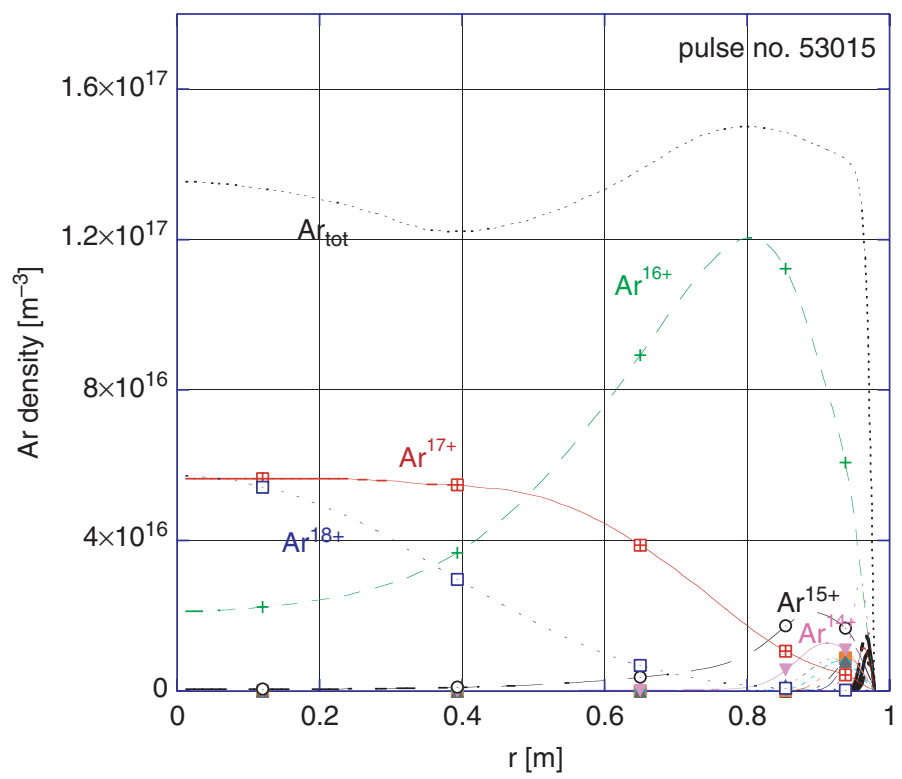

Figure 6. Same as figure 4 for \#53015 at $t=2 \mathrm{~s}$ after the start of the AP phase, to be associated to the radiated power density profile shown in figure 5 . 
of a test Ar ion (e.g. He-like $\mathrm{Ar}^{16+}$ ) is expressed as the sum of diffusive and convective terms, which include both the Pfirsh-Schluter plus classical (PS) and banana-plateau (BP) terms:

$\Gamma_{\mathrm{ncl}}=\Gamma_{\mathrm{BP}}+\Gamma_{\mathrm{PS}}=-\left(D_{\mathrm{BP}}+D_{\mathrm{PS}}\right) \frac{\partial n_{Z}}{\partial r}+\left(v_{\mathrm{BP}}+v_{\mathrm{PS}}\right) n_{Z}=-D_{\mathrm{ncl}} \frac{\partial n_{Z}}{\partial r}+v_{\mathrm{ncl}} n_{Z}$.

Since the neoclassical coefficients depend not only on the electron density and temperature, but also on the ion distribution, the ion density profiles obtained as output of the simulation have been used for their calculation. The BP coefficients have been evaluated following [11], and the PS according to [12]; the effect of the collisions of the test ions with D, C and the other Ar ions is also taken into account. The result of the calculation is not considered to be reliable in the external plasma region ( $r / a>0.8$, where $a$ is the LCFS radius), since the uncertainty associated to the gradients (essentially of the ion distribution and also of temperature and density) is large. The peaking factor $v_{\text {ncl }} / D_{\text {ncl }}$ obtained from the neoclassical calculation around one-half of the plasma radius is about $-20 \mathrm{~m}^{-1}$, to be compared with a positive and much lower value $\left(\sim 0.1 \mathrm{~m}^{-1}\right)$ deduced from the simulation. The reason of this anomaly is due to the fact that the neoclassical drift velocity is found to be inwards, and the diffusion coefficient from the neoclassical theory is about $0.05-0.1 \mathrm{~m}^{2} \mathrm{~s}^{-1}$, i.e. lower than that found from the simulation by a factor of about 5 . It can be observed that for the previous shot \#52136 the simulation gives a peaking factor of about $-4 \mathrm{~m}^{-1}$, something closer to the neoclassical one, that results to be of the order of $-20 \mathrm{~m}^{-1}$ also in this case. It must be finally mentioned that for a fully consistent comparison, the simulation should be repeated with the neoclassical transport coefficients, comparing the resulting ion densities. Here a simplified approach has been used to have an order-of-magnitude comparison with the neoclassical diffusion coefficients and convective velocities.

\subsection{Very high triangularity discharge with continuous $D_{2}$ puffing and $12 \mathrm{MW}$ NBI plus 1.7 MW ICRH power}

Of particular interest is the analysis of very high triangularity discharges, such as shot \#53548, in which argon has been injected as short 'blips' during a continuous deuterium puffing. In these discharges a relatively stationary phase is observed (about $3 \mathrm{~s}$ for shot \#53548) with $\gamma_{\text {RAD }}>60 \%$ and hollow radiation profiles, as shown in figure 7 . During this phase a considerably high radiation at the edge is obtained without a severe increase in the centre.

In this pulse a higher $(\sim 5) \rho$-ratio between the $\mathrm{C}$ vi Ly $\alpha$ and $\mathrm{C} v \mathrm{R}$ lines has been observed. This ratio depends on the ionization degree of the $\mathrm{C}$ ions around the LCFS. It is well-known (see, e.g. [13]) that the latter is affected by CX recombination processes of $\mathrm{C}$ ions with neutral hydrogen isotopes. Moreover, the G-ratio of the two $\mathrm{Cv}$ intercombination and resonance lines is an increasing function of the neutral density, besides being an inverse function of the electron temperature. To simulate these $\mathrm{C}$ line ratios, it was necessary to postulate a neutral edge density of $7 \times 10^{15} \mathrm{~m}^{-3}$ (of the order of $0.01 \%$ of the on-axis electron density). Direct absolute measurements of the neutral density are not available, but a significant presence of neutrals at the edge is consistent with the high $\mathrm{D}_{2}$ influx puffed into the plasma and with an higher experimental level of the $\mathrm{D}_{\alpha}$ emission from visible spectroscopy (by a factor of about 3 in-between ELMs) and of the Ly $\beta$ emission from the Spred spectrum (again a factor of 2-3) in comparison with the previously analysed discharges. With this neutral density (that is of the same order as found on the equatorial plane in DIII-D L-mode divertor discharges [14]), the $\rho$-ratio during the AP increases up to 4 (the value without neutrals would be 2.1), that is still about $20 \%$ lower than the experimental; the G-ratio results to be 0.4 , in agreement with the measured spectrum. 


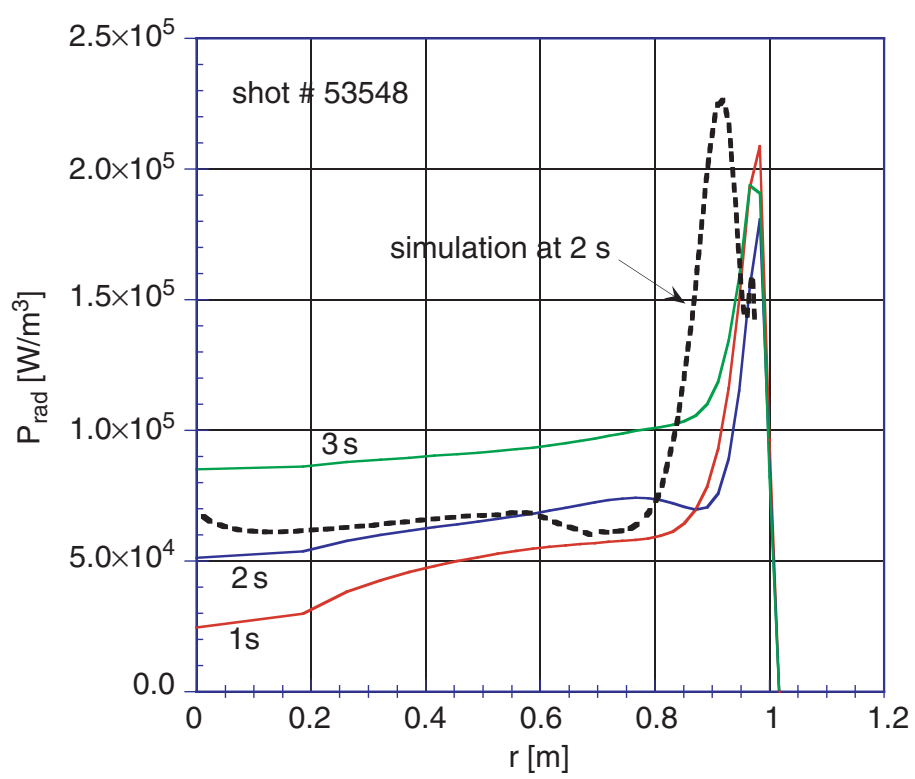

Figure 7. Same as figure 2 during the AP phase (starting for this shot at the beginning of the Ar blips) of pulse \#53548 (very high triangularity discharge).

To be consistent, the contribution of $\mathrm{CX}$ recombination for $\mathrm{Ar}$ has been included as well. The used CX cross sections have been discussed in [4]. The radiation loss due to deuterium (following [15] ${ }^{11}$ ) has also been added to the radiated power calculation, resulting to be negligible.

The transport parameters that allow the simulation of the impurity behaviour are a pinch velocity outward (and low) in the central half of the plasma, and inward in the external half (figure 3), with a diffusion coefficient only slightly higher than for the previous discharges in the external half of the radius, inside the diffusive barrier $\left(1 \mathrm{~m}^{2} \mathrm{~s}^{-1}\right)$. As an example, figure 8 shows the time evolution of the ArXVI and of the SXR brightnesses measured along a central chord compared with the simulation. The SXR brightnesses in the figure are normalized; however, in absolute units the calculated value is $2.5 \times 10^{4} \mathrm{~W} \mathrm{~m}^{-2}$ at $t=2 \mathrm{~s}$ after the start of argon puff, to be compared with an experimental one of $3 \times 10^{4}$ (measured on one of the central chords). The radiated power profile is reconstructed as shown in figure 7 and is characterized by a high edge radiation. When compared with the previously reported discharge \#53015, that also showed an hollow radiation profile in the central AP phase (at $2 \mathrm{~s}$ ), the radiation enhancement is more localized at the edge, and its maximum value is higher. The corresponding total $\mathrm{Ar}$ ion density (figure 9) is flat, similarly to that of shot \#53015, but the presence of neutrals at the very edge reduces, via the charge-exchange processes, the average degree of ionization of $\mathrm{Ar}$ at the edge, thus contributing to the enhancement of the edge radiation. Of course, due to charge-exchange processes, also the contribution of carbon to the radiated power, that is not negligible at the edge, increases. Indeed the inclusion of neutrals in the simulation implies an increase of $\sim 30-40 \%$ in the maximum value of the edge radiated power density.

As previously, the neoclassical calculations give an inward velocity, with a peaking factor of about $-10 \mathrm{~m}^{-1}$ in the central plasma, while the low outward velocity used for the simulation

${ }^{11}$ Revised data kindly made available by R Marchand. 

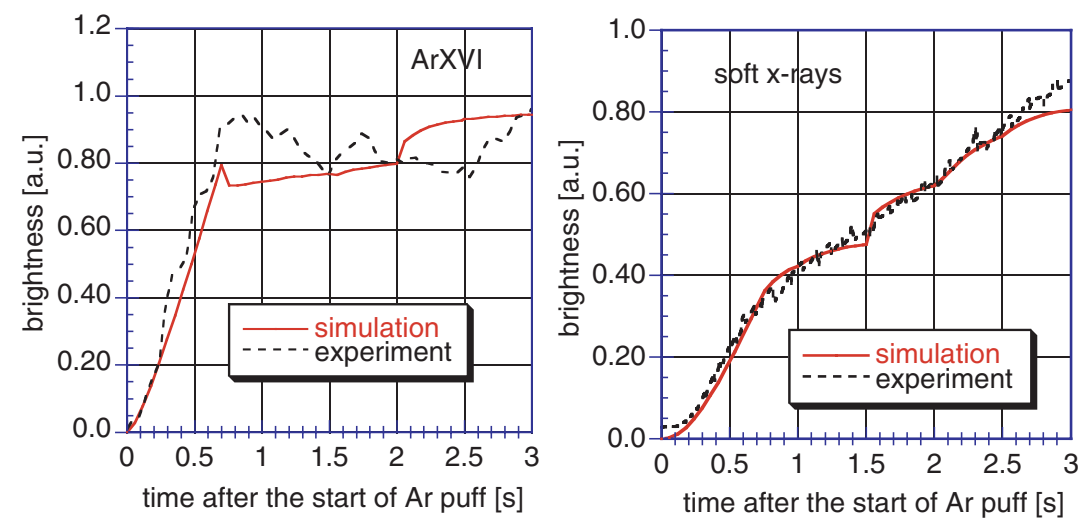

Figure 8. Comparison between the experimental and simulated time evolution of the Ar Xvi $353 \AA$ line brightness (normalized) and the soft X-ray brightness (normalized) in pulse \#53548. In this case times are referred to the start of the argon inflow phase.

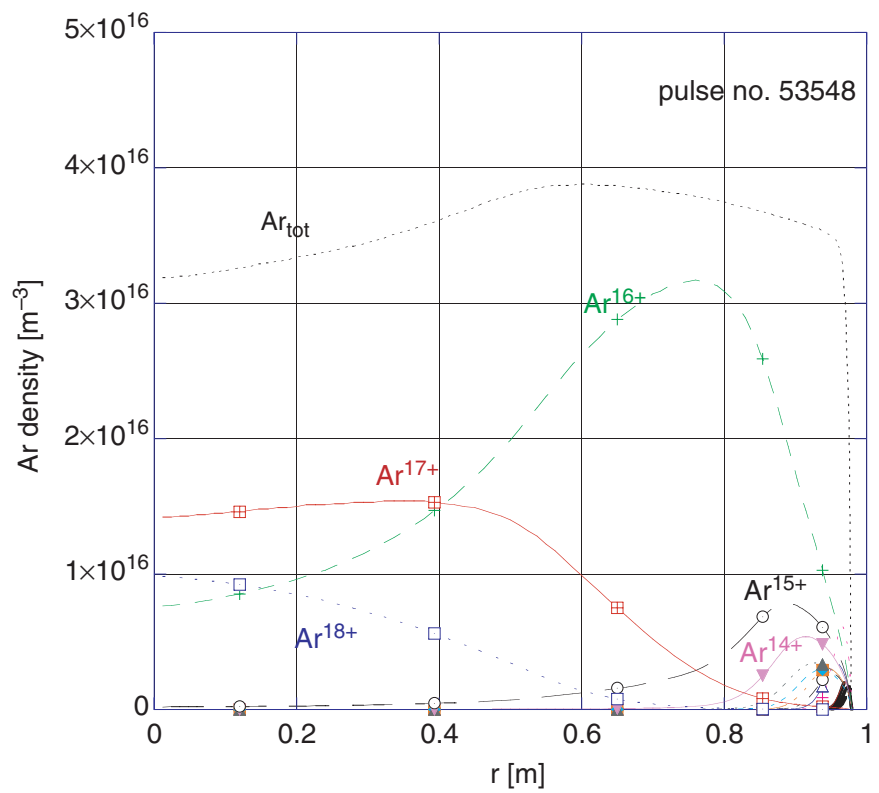

Figure 9. Same as figure 4 for $\# 53548$ at $t=2 \mathrm{~s}$ after the start of the AP phase. At the edge, low-charge highly radiative ions are more populated than in the previously analysed discharges.

gives a very low positive $(v / D)$-ratio $\left(\sim 0.1 \mathrm{~m}^{-1}\right)$.

Figure 10 summarizes the analysed experimental scenarios in terms of the two 'macroscopic' parameters showing the impurity behaviour: the radiated power density (experimental in figure $10(a)$ ) and the effective charge (simulated in figure $10(b)$ ). Their profiles at $t=2 \mathrm{~s}$ are shown as a function of the radius for three discharges. It can be observed that the high triangularity divertor discharge corresponds to the best situation: the effective charge is low in the centre and the radiation increases strongly in a narrow region at the edge: a radiative mantle without strong contamination of the plasma centre is achieved. 

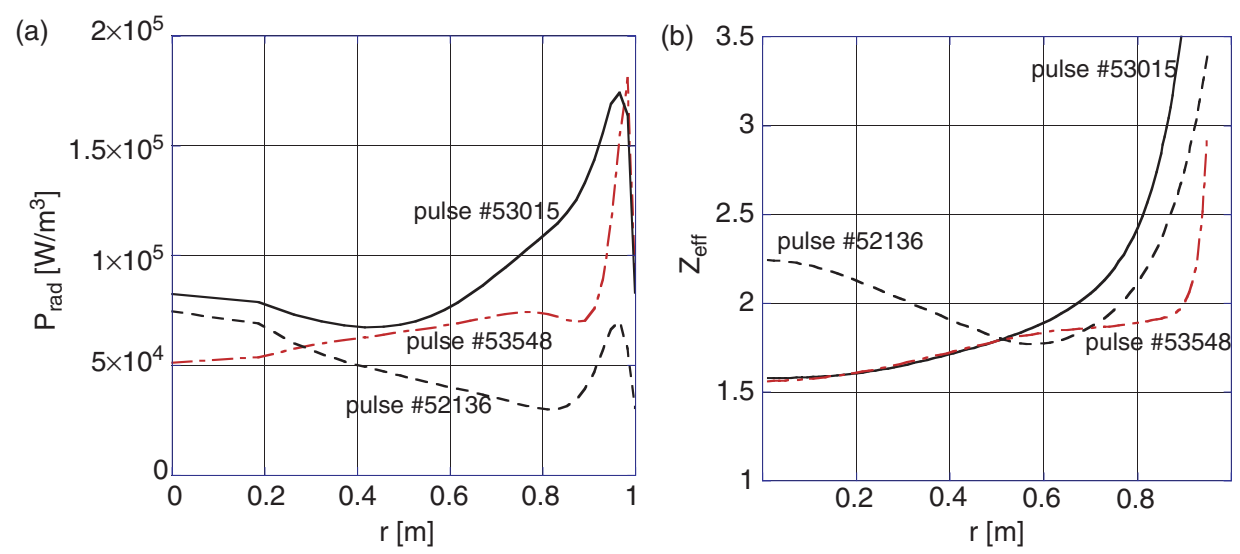

Figure 10. Radial profiles of $(a)$ the Abel-inverted experimental radiated power density profiles and $(b)$ the effective charge $Z_{\text {eff }}$ as obtained from the simulations for the three analysed discharges, always $2 \mathrm{~s}$ after the beginning of the AP phase.

\section{Argon spectrum simulations}

The simulation of the emission spectra in the XUV and VUV spectral ranges has been performed including full spectral simulation of $\operatorname{Ar} n=2$ to $n=3$ and $n=2$ to $n=2 \mathrm{~L}$-shell, in the same way as previously reported in $[3,4]$. The line brightnesses have been evaluated in a post-processing subroutine which simulates the spectra. For this purpose, collisional-radiative (CR) models have been built for the ions whose lines are observed in the spectra, considering electron collisional excitation and radiative decay as the populating processes of the excited states. These models give photon emission coefficients (PECs) for the emitted lines at electron density and temperature values corresponding to the experimental situations.

While the spectra corresponding to a single ionization state have a weak dependence on the experimental conditions, the superposition of these partial spectra depends on the ion charge distribution, which in turn depends on the radial profiles of the electron density and temperature and on the impurity transport coefficients. The simulation of the spectra allows the determination of the two transport coefficients in the plasma region emitting the considered ionization states, that corresponds to the region just inside the LCFS. By the way, the analysis of spectra emitted by hot and well diagnosed magnetic fusion confinement plasmas touches atomic physics issues that are required for simulation codes to be used in the analysis of high spectral resolution data from x-ray telescopes [16].

As discussed in $[3,4]$ the atomic coefficients used for this purpose for argon have been calculated by the Hebrew University Lawrence Livermore Atomic Codes HULLAC, while for the $\mathrm{C}$ vi and $\mathrm{C} v$ lines the coefficients from [13] have been used. Figure 11 shows the result obtained for the high triangularity discharge $\# 53548$, respectively, for the spectra obtained with the XUV $(a)$ and the VUV $(b)$ spectrometers at $t=2 \mathrm{~s}$ during the AP phase. A calibration curve of the XUV spectrometer is not available; therefore, the sensitivity has been assumed as uniform in figure 11(a); however, it has to be mentioned that the calibration of similar instruments [17] shows an increasing efficiency with the wavelength between 25 and $40 \AA$ As As a consequence, in particular the ratio between the $\mathrm{C}$ VI and $\mathrm{C} v$ resonant lines, that experimentally is already higher than in the simulation, could be even further underestimated (up to a factor 1.5). An ion temperature somewhat higher in the region where $\mathrm{C}$ VI and $\mathrm{C} v$ lines are emitted (in absence of edge ion temperature measurements in the simulation $T_{\mathrm{I}}=T_{\mathrm{e}}$ has been assumed) could 

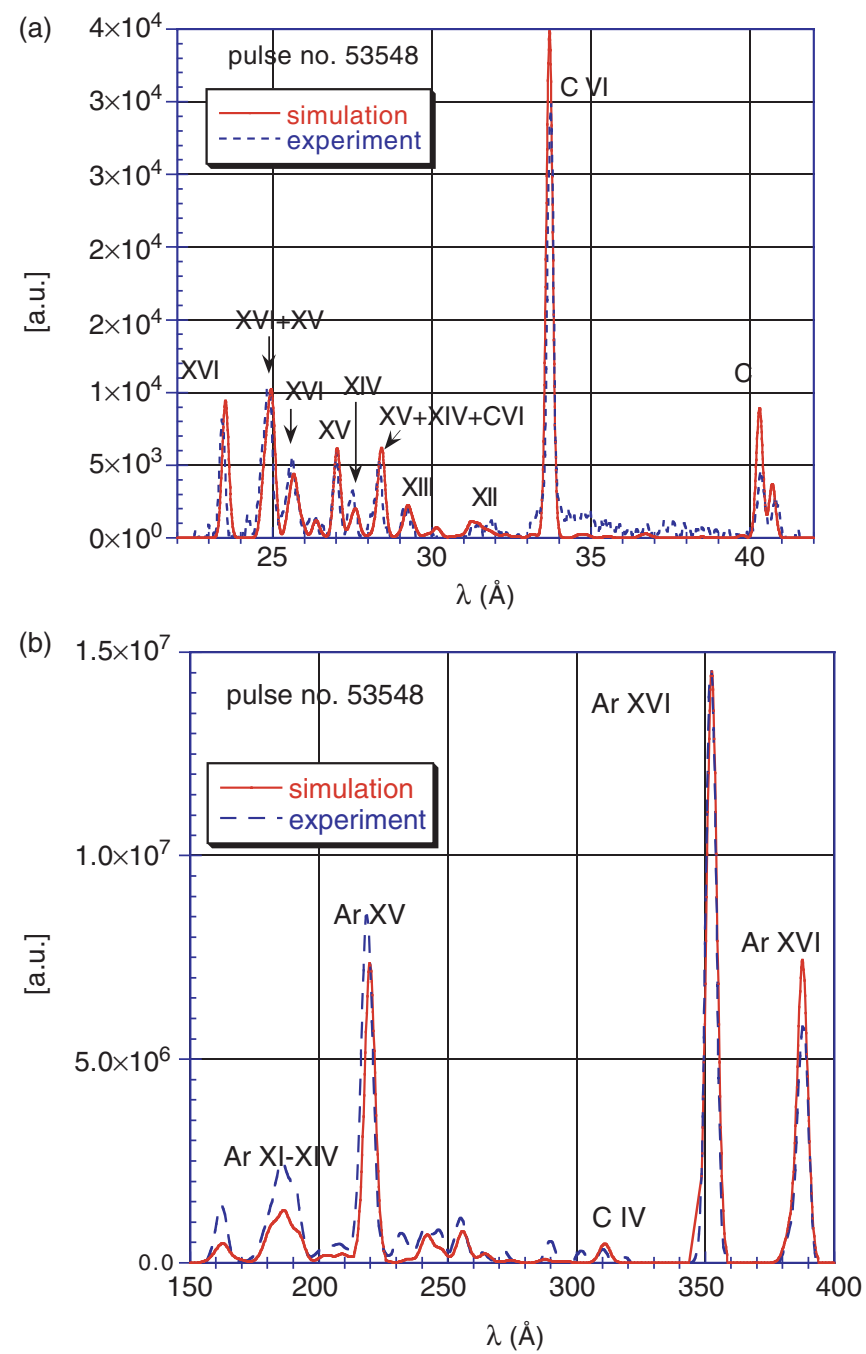

Figure 11. Simulation of the XUV $(a)$ and VUV $(b)$ spectra in pulse \#53548 during the AP phase. The y axis, in arbitrary units, corresponds to counts/s.

partly compensate for this discrepancy; indeed a higher $T_{\mathrm{I}}$ would imply higher CX rates. In figure 11(b) the simulation is not as good as that reported in [4] for the secondary peaks; an explanation may be that the calculated spectrum does not take into account some weak lines from Ni (165, 234 and $292 \AA$ ) and (possibly) O (150 and $173 \AA$ ).

To obtain agreement of the Ar spectrum simulations both $D(r)$ and $v(r)$ have been shaped to produce an edge peripheral barrier (either decreasing $D$ or increasing $v$, or both simultaneously), as previously found in Ar injection JET data analysis [3,4]. The uncertainty in some edge parameters (neutral deuterium density, electron temperature and density) prevents to define quantitatively this edge barrier and therefore to reach a unique final choice for the transport coefficients. 


\section{Conclusions}

The radiation behaviour and impurity transport in the high performance argon seeded JET discharges have been analysed, in order to understand if high radiation from the plasma edge can be achieved without heavy contamination of the plasma centre.

In the first experimental configuration (described in section 3.2), in a septum low triangularity plasma heated by $12 \mathrm{MW}$ of NBI, in spite of enhanced confinement during the AP phase, central Ar ion accumulation occurs, whereas $\mathrm{C}$ ions keep a hollow profile. On the other hand, two configurations (described in sections 3.3 and 3.4) with enhanced confinement during the AP phase have been obtained avoiding impurity accumulation in the plasma centre. In the first one a moderate amount of ICRH central heating ( $2 \mathrm{MW})$ is applied in a septum low triangularity plasma on top of $12 \mathrm{MW}$ NBI, with a beneficial effect on preventing the central plasma cooling and maintaining the sawtooth; the simulation indicates a low pinch Ar ion velocity in the centre, outwards only in a first phase of the AP and then inwards, that favours the formation of hollow or flat profiles of Ar ions. In this case the radiated power density profile is always hollow, but still with a high level from the centre, and a quite wide external highly radiative region $(r / a \geqslant 0.6)$. In the second example, at very high triangularity with a continuous $\mathrm{D}_{2}$ puffing, in which again a central pinch Ar ion velocity outward directed has been found to simulate the impurity behaviour, the radiation profiles are hollow with a narrow highly radiative region well localized at the plasma edge, and lower radiation from the centre. In this discharge, the effective charge remains low $(\sim 1.5)$ in the centre. It has been found some evidence from the spectral simulations that this behaviour can be associated to the contribution of charge exchange processes, which favour the population of highly radiative charge states at the edge.

In both these cases, the comparison with the neoclassical theory has shown a significant anomalous contribution to the diffusion coefficient, whereas, differently from the values found with the simulations, the neoclassical velocity results to be always inwards.

Among the three analysed discharges, with the third one, performed at high triangularity with 1.7 MW ICRH added to NBI, a radiative mantle has been achieved, maintaining a highdensity, high-confinement plasma without severe increase of both radiation and effective charge in the centre. It is still a matter of discussion if this effect has to be associated either to the ST maintainance due to ICRH or to the high triangularity configuration or if it derives from a combination of these two causes.

\section{References}

[1] Ongena J et al 2001 Phys. Plasmas 82188

[2] Dumortier P et al 2001 28th EPS Conf. on Control. Fusion and Plasma Phys. (Funchal, 18-22 June 2001) vol 25A, p 941

[3] Mattioli M et al 2001 J. Phys. B: At. Mol. Opt. Phys. 34127

[4] Mattioli M et al 2002 Plasma Phys. Control. Fusion 4433

[5] Nave M F F et al 2001 28th EPS Conf. on Control. Fusion and Plasma Phys. (Funchal, 18-22 June 2001) vol 25A, p 961

[6] Pasini D et al 1992 Plasma Phys. Control. Fusion 34677

[7] Giannella R et al 1994 Nucl. Fusion 341185

[8] Chen H et al 2001 Nucl. Fusion 4131

[9] Schwob J L, Wouters A W, Suckever S and Finkenthal M 1987 Rev. Sci. Instrum. 581601

[10] Fonck R J, Ramsey A T and Yelle R W 1982 Appl. Opt. 212115

[11] Hawryluck R, Suckewer S and Hirshman S P 1979 Nucl. Fusion 19607

[12] Hirshman S P and Sigmar P J 1981 Nucl. Fusion 211079

[13] Carraro L et al 1997 Phys. Scr. 55565 
[14] Colchin R J et al 2000 27th EPS Conf. on Control. Fusion and Plasma Phys. (Budapest) vol 24B, p 760

[15] Bonnin X, Marchand R and Janev R K 1992 Atomic and plasma-material interaction data Nucl. Fusion 2 (suppl) 117

[16] Fournier K B et al 2000 Proc. Workshop on Atomic Data Needs for X-Ray astronomy ed M Bautista, T Kallman and A Pradhan, vol 1 (Washington, DC: NASA Publications) p 127 (also http://heasarc.gsfc.nasa.gov/docs/heasarc/atomic/)

[17] Carraro L, Puiatti M E, Scarin P and Valisa M 1995 Rev. Sci. Instrum. 66613 